\title{
Active mechanistic target of rapamycin plays an ancillary rather than essential role in zebrafish CNS axon regeneration
}

\author{
Heike Diekmann, Pascal Kalbhen and Dietmar Fischer* \\ Division of Experimental Neurology, Department of Neurology, Heinrich-Heine-University of Düsseldorf, Düsseldorf, Germany
}

The developmental decrease of the intrinsic regenerative ability of the mammalian central nervous system (CNS) is associated with reduced activity of mechanistic target of rapamycin (mTOR) in mature neurons such as retinal ganglion cells (RGCs). While mTOR activity is further decreased upon axonal injury, maintenance of its pre-injury level, for instance by genetic deletion of the phosphatase and tensin homolog (PTEN), markedly promotes axon regeneration in mammals. The current study now addressed the question whether active mTOR might generally play a central role in axon regeneration by analyzing its requirement in regeneration-competent zebrafish. Remarkably, regulation

OPEN ACCESS

Edited by:

Andrea Nistri,

Scuola Internazionale Superiore

di Studi Avanzati, Italy

Reviewed by:

Xiao-Feng Zhao,

University of Michigan, USA Miranda Mladinic,

University of Rijeka, Croatia

*Correspondence: Dietmar Fischer,

Division of Experimental Neurology, Department of Neurology, Heinrich-Heine-University of Düsseldorf, Merowingerplatz 1a, 40225 Düsseldorf, Germany dietmar.fischer@uni-duesseldorf.de

Received: 04 June 2015 Accepted: 22 June 2015 Published: 07 July 2015

Citation:

Diekmann $\mathrm{H}$, Kalbhen $\mathrm{P}$ and Fischer D (2015) Active mechanistic target of rapamycin plays an ancillary rather than essential role in zebrafish

CNS axon regeneration.

Front. Cell. Neurosci. 9:251. doi: 10.3389/fncel.2015.00251 of mTOR activity after optic nerve injury in zebrafish is fundamentally different compared to mammals. Hardly any activity was detected in naïve RGCs, whereas it was markedly increased upon axotomy in vivo as well as in dissociated cell cultures. After a short burst, mTOR activity was quickly attenuated, which is contrary to the requirements for axon regeneration in mammals. Surprisingly, mTOR activity was not essential for axonal growth per se, but correlated with cytokine- and PTEN inhibitor-induced neurite extension in vitro. Moreover, inhibition of mTOR using rapamycin significantly reduced axon regeneration in vivo and compromised functional recovery after optic nerve injury. Therefore, axotomy-induced mTOR activity is involved in CNS axon regeneration in zebrafish similar to mammals, although it plays an ancillary rather than essential role in this regeneration-competent species.

\section{Keywords: mTOR, optic nerve regeneration, zebrafish, rapamycin, pS6}

\section{Introduction}

The regenerative ability of the mammalian central nervous system (CNS) is dramatically reduced after birth, causing impaired regeneration of severed axons in adult animals. This regenerative failure is partly based on the limited intrinsic regenerative ability of mammalian CNS neurons and the inhibitory environment of axonal growth cones

\footnotetext{
Abbreviations: 4E-BP1, 4E-binding protein 1; AKT, protein kinase B; bpV, bisperoxovanadium; CHAT, cholinergic acetyl transferase; CNTF, ciliary neurotrophic factor; dpi, day(s) post injury; DRG, dorsal root ganglion; GAP43, growth associated protein 43; GFP, green fluorescent protein; JAK, Janus kinase; LIF, leukemia inhibitory factor; mTor, mechanistic target of rapamycin; PI3K, phosphatidylinositol 3-kinase; PTEN, phosphatase and tensin homolog; RGC, retinal ganglion cell; S6, ribosomal protein S6; S6K1, ribosomal protein S6 kinase 1; STAT, signal transducer and activator of transcription; TSC1, tuberous sclerosis complex 1.
} 
(Schwab and Bartholdi, 1996; Goldberg et al., 2002; Fischer and Leibinger, 2012). However, some degree of axonal regeneration is observed upon induction of cytokine expression by inflammatory stimulation (Fischer et al., 2000; Leibinger et al., 2009, 2013; Hauk et al., 2010), intravitreal application of ciliary neurotrophic factor (CNTF) (Lingor et al., 2008; Müller et al., 2009) and virusmediated cytokine expression (Leaver et al., 2006; Pernet et al., 2013). In addition, genetic manipulation of certain signaling cascades, such as phosphatidylinositol 3-kinase/protein kinase $\mathrm{B} /$ mechanistic target of rapamycin (PI3K/AKT/mTOR) and Janus kinase/signal transducer and activator of transcription (JAK/STAT) have been described to promote axon regeneration (Cai et al., 2001; Gao et al., 2004; Fischer and Leibinger, 2012; Diekmann and Fischer, 2013; Pernet et al., 2013). In particular, genetic deletion of the phosphatase and tensin homolog (PTEN), an opponent of PI3K, potently promotes axon regeneration of RGCs, motor, corticospinal and peripheral neurons (Park et al., 2008; Christie et al., 2010; Liu et al., 2010; Ning et al., 2010). In RGCs, these effects were, for the most part, mimicked by knockout of tuberous sclerosis complex 1 (TSC1), a negative regulator of mTOR signaling (Park et al., 2008). Therefore, the growth promoting effects of PTEN deletion have been mainly attributed to sustained activity of downstream mTOR. This serine-threonine protein kinase is central to a multi-functional signaling network that integrates intra- and extracellular signals to control cell proliferation, metabolism and survival (Maiese et al., 2013). Upon nucleation into multi-protein complexes, mTOR phosphorylates, among others, ribosomal protein S6 kinase 1 (S6K1) and eukaryotic initiation factor 4E-binding protein 1 (4E-BP1), thereby promoting anabolic processes such as protein and lipid synthesis (Laplante and Sabatini, 2009). Inhibition of mTOR by rapamycin attenuates most of the beneficial effects of PTEN deletion in RGCs, compromises optic nerve regeneration upon inflammatory stimulation and partially blocks the conditioning effect of sciatic nerve injury (Park et al., 2008; Abe et al., 2010; Leibinger et al., 2012). Therefore, these findings suggest that mTOR plays a central role in regenerative processes of injured axons in mammals and raise the question as to whether mTOR activity is also required for axon growth stimulation in regeneration-competent animals.

In contrast to mammals, adult teleosts have the remarkable ability to functionally regenerate injured CNS axons (Stuermer et al., 1992; Bernhardt et al., 1996; Becker and Becker, 2007). Upon crush or complete transection of the optic nerve, RGCs survive and regrow axons through the optic nerve and tract to topographically re-innervate their respective targets in the brain, leading to functional restoration of the visual projection within 2-4 weeks after injury (Stuermer et al., 1992; Bernhardt, 1999; McDowell et al., 2004). We recently established the adult transgenic growth associated protein 43 (GAP43):green fluorescent protein (GFP) zebrafish as a valuable tool for axon regeneration studies, as they strongly express GFP only in injured neurons and their regrowing axons (Diekmann et al., 2015). In addition, the analysis of regenerating axons was facilitated using a combination of wholemount optic nerve tissue clearing and confocal microscopy (Diekmann et al., 2015). Taking advantage of these novel approaches, we here investigated the regulation of mTOR after optic nerve injury in adult zebrafish and whether manipulation of mTOR activity would impact RGC axon regeneration in vitro and in vivo. Indeed, we detected pronounced, although transient increase of mTOR activity in most RGCs upon optic nerve injury in vivo as well as in dissociated cell cultures. Moreover, inhibition of mTOR attenuated CNTF- and PTEN inhibitorinduced, but not basal axonal growth in vitro as well as axon regeneration in vivo. In addition, functional recovery in vivo was significantly compromised upon rapamycin treatment, indicating a contribution of mTOR signaling to spontaneous axon regeneration in zebrafish.

\section{Materials and Methods}

\section{Zebrafish}

Adult, 4-9 months old wild-type or homozygous $\mathrm{Tg}$ (GAP43:GFP) zebrafish (Udvadia, 2008), in the text referred to as GAP43:GFP, were used for all experiments. Zebrafish were reared and kept on a $14 \mathrm{~h}$ light/ $10 \mathrm{~h}$ dark cycle under standard conditions (Westerfield, 1989). All experimental procedures were approved by the local animal welfare committee in Recklinghausen and conducted in compliance with federal and state guidelines for animal experiments in Germany.

\section{Dissociated Retinal Cell Cultures}

Tissue culture plates (24- or 96-well-plates; Nunc) were coated with poly-D-Lysine $(0.1 \mathrm{mg} / \mathrm{ml}$, molecular weight $3,00,000$ Da; Sigma) for $30 \mathrm{~min}$ at $37^{\circ} \mathrm{C}$, rinsed twice with distilled water and air-dried. Zebrafish were sacrificed by immersion in MS222 $(0.4 \mathrm{mg} / \mathrm{l})$ and decapitation. Retinae were rapidly dissected from the eyecups of naive zebrafish and incubated in a digestion solution containing papain $(10 \mathrm{U} / \mathrm{ml}$, Worthington) and L-cysteine $(0.3 \mu \mathrm{g} / \mathrm{ml}$; Sigma) in L15/salt solution (12.5\% salt solution: $10 \mathrm{mM}$ D-glucose, $1.26 \mathrm{mM} \mathrm{CaCl}_{2}, 32 \mathrm{mM}$ Hepes, $\mathrm{pH}$ 7.5/ 87.5\% L15; Invitrogen) at room temperature for $40 \mathrm{~min}$. They were then rinsed with L15/salt solution and triturated in $2 \mathrm{ml}$ fish medium (2\% FBS (Invitrogen), $0.2 \mathrm{mg} / \mathrm{ml} \mathrm{penicillin/streptomycin} \mathrm{(Biochrom)} \mathrm{in} \mathrm{L15/salt}$ solution). Dissociated cells were passed through a cell strainer (40 $\mu \mathrm{m}$; Falcon) and counted using a TC10 Automated Cell Counter (BioRad). Approximately $1.5 \times 10^{4}$ cells (96 well plate) and $2.5 \times 10^{4}$ cells (24 well plate) were added to each well, respectively. In some wells, $1 \mathrm{ng} / \mathrm{ml}$ mouse CNTF (Peprotech), $10 \mathrm{nM}$ rapamycin (LC Laboratories) or $10 \mathrm{nM}$ bisperoxovanadium (bpV(phen), Calbiochem) were added to the culture medium. Cultures were incubated at $27.5^{\circ} \mathrm{C}$ in a humidified incubator for $2 \mathrm{~h}$ to 4 days. Cells were fixed with $4 \%$ paraformaldehyde (PFA) in phosphate-buffered saline (PBS) for $30 \mathrm{~min}$ at room temperature and permeabilized with Methanol (5 min) for immunostaining. Neurite growth was determined after 4 days in culture. RGCs with regenerated neurites were visualized via GFP expression or acetylated tubulin antibody (1:2,000; Sigma) immunostaining and photographed under a fluorescent microscope (200×, Observer.D1, Zeiss). Neurite length was determined using ImageJ software as previously described (Diekmann et al., 2015). Mean neurite length was 
calculated by dividing the sum of neurite length by the number of RGCs with regenerated neurites per well. Data are given as means \pm SEM of at least six replicate wells from at least two independent experiments. The significance of intergroup differences was evaluated using Two-Way Anova with HolmSidak post hoc tests (GraphPad; SigmaStat).

\section{Optic Nerve Crush}

For surgery, zebrafish were anesthetized by immersion in MS222 $(0.18 \mathrm{mg} / \mathrm{l}$; Sigma). The eye was slightly pulled out of its orbit to expose the optic nerve. Taking care to spare the ophthalmic artery, the optic nerve was intra-orbitally crushed $\sim 0.5 \mathrm{~mm}$ behind the eye for $5 \mathrm{~s}$ using jeweler's forceps (FST), as described previously (Bormann et al., 1999; Liu and Londraville, 2003; Diekmann et al., 2015).

\section{In vivo Rapamycin Treatment}

A rapamycin stock of $1 \mathrm{mg} / \mathrm{ml}$ was prepared in DMSO. Immediately after optic nerve crush, fish were placed into $0.2 \mu \mathrm{M}$ rapamycin (1:5,000 dilution of stock in fish water) or $0.02 \%$ DMSO as control. Solutions were changed daily.

\section{Retinal Cross Sections}

Zebrafish were sacrificed by prolonged immersion in MS222 (0.4 $\mathrm{mg} / \mathrm{l})$ and decapitation. Eye(s) were removed and fixed in $4 \%$ PFA/PBS at $4^{\circ} \mathrm{C}$ overnight. Subsequently, eyes were immersed in $30 \%$ sucrose and embedded in Tissue-Tek (Sakura). Frozen sections $(14 \mu \mathrm{m})$ were cut on a CM3050S cryostat (Leica), thawmounted onto glass slides (Superfrost plus; ThermoFisher) and stored at $-80^{\circ} \mathrm{C}$ until further use.

\section{Immunohistochemistry}

Retinal cross sections were permeabilized with 100\% Methanol for $5 \mathrm{~min}$ at room temperature. After blocking with $2 \%$ $\mathrm{BSA} / 5 \%$ donkey serum/PBS, they were incubated with either pS6(Ser235/236) (1:500; Cell Signaling \#4857), choline acetyl transferase (CHAT; 1:100; Millipore AB144P) or acetylated tubulin (1:1,000; Sigma T6793) antibodies overnight at $4^{\circ} \mathrm{C}$. The protein sequence of zebrafish ribosomal S6 around the detected phosphorylation site is $100 \%$ identical to the human peptide used for antibody generation. After several washes with PBS, bound antibodies were visualized with anti-mouse or anti-goat secondary antibodies conjugated to Alexa Fluor 488 or Alexa Fluor 594 (1:1,000; Molecular Probes). Fluorescent sections were embedded in Mowiol and analyzed under a fluorescent microscope $(200 \times$, Observer.D1, Zeiss). The percentage of pS6-positive cells was calculated in relation to the number of cells stained with acetylated tubulin in the ganglion cell layer (RGCs) and CHAT-positive cells in the inner nuclear layer (INL; amacrines), respectively, on six sections from 2-3 different zebrafish at each time point.

\section{Quantification of Axon Regeneration}

Two and a half days after optic nerve crush, zebrafish were sacrificed by prolonged immersion in MS222 $(0.4 \mathrm{mg} / \mathrm{l})$ and decapitation. The lower jaw and gills were removed and the eyes pulled slightly out of their sockets to stretch the optic nerves. The head was then fixed in $75 \mathrm{mM}$ Lysine/2\% PFA/10 mM NaIO overnight at $4^{\circ} \mathrm{C}$. After fixation, the optic nerves were dissected with part of the retina attached and placed into FocusClear solution (BioRad) overnight for clearing. They were embedded in MountClear and scanned using a confocal microscope (LSM510, Zeiss). For quantification of axon regeneration, axon profiles within the nerve diameter were counted on five individual z-sections at 200 and $500 \mu \mathrm{m}$ distal from the lesion site, respectively. "Axons per $\mathrm{mm}$ " was calculated by dividing the number of axons per site by the diameter of the nerve of the respective $\mathrm{z}$-section. Data are given as means \pm SEM of eight optic nerves per group from two independent experiments. The significance of intergroup differences was evaluated using Two-Way ANOVA with Holm-Sidak post hoc tests (GraphPad; SigmaStat).

\section{Dorsal Light Reaction}

Fish swim in a slightly oblique position $\left(\sim 10^{\circ}\right)$ upon unilateral optic nerve injury (Figure 5E; Lindsey and Powers, 2007; Mensinger and Powers, 2007), which is gradually reversed with ongoing regeneration. Therefore, the degree of tilt can serve as a measure for functional regeneration. At various times after optic nerve injury, fish were placed into a $2.7 \times 17.5 \mathrm{~cm}$ container with $400 \mathrm{ml}$ water. After $\sim 5$ min adaptation, they were recorded on video for 1-2 min, making sure to capture at least five straight swims directly towards the camera. The videos were analyzed frame by frame and still pictures taken if the whole body of the fish was positioned straight towards the camera. The angle between the fish body position (straight line through the eyes; Figure 5E) and the horizon was then determined using ImageJ. At least seven different pictures were analyzed per fish and time point to calculate the mean divergent angle. Data are given as means \pm SEM of at least five fish per group. The significance of intergroup differences was evaluated using Repeat-Measurements TwoWay ANOVA with Holm-Sidak post hoc tests (GraphPad; SigmaStat).

\section{RNA Isolation and Quantitative Real-Time PCR}

For quantitative real-time PCR, both retinae from one zebrafish were dissected and combined. Total RNA was isolated using the RNeasy Mini kit (Qiagen, Germany) according to the manufacturer's instructions, including the removal of genomic DNA. Total RNA (300 ng) was reverse transcribed using the SuperScript II reverse transcriptase kit (Invitrogen, USA). Gfp, gap43 and glyceraldehyde 3-phosphate dehydrogenase (gapdh) were amplified using SYBR Green PCR Master Mix (Applied Biosystems, Foster City, CA, USA) and primers gfp-for (5'-GCAAGCTGACCCTGAAGTTC-3'), gfp-rev (5'-GGTGCGCTCCTGGACGTA-3'), gap43-for $\left(5^{\prime}\right.$ TGCTGCATCAGAAGAACTAA-3'), gap43-rev (5'-CCTCCG GTTTGATTCCATC-3'), gapdh-for (5'-CCTCCGGTTTGAT TCCATC-3') and gapdh-rev (5'-GGCGGTGTAGGCATGAAC$\left.3^{\prime}\right)$ on an Applied Biosystems 7500 real-time PCR system (Life Technologies) using 45 amplification cycles according to the manufacturer's protocol. PCR efficiency was confirmed 
for each primer pair and specificity verified with the dissociation curve analysis feature. Gfp and gap43 mRNA expression levels in rapamycin-treated vs. control retinae were quantified using the $\Delta \Delta \mathrm{Ct}$ method (Livak and Schmittgen, 2001) with gapdh as the reference gene. All reactions were performed in triplicate and at least three independent samples (from different zebrafish) were analyzed per experimental group.

\section{Results}

\section{Optic Nerve Injury Induces mTOR Activity in Zebrafish Retinae}

In order to analyze mTOR activity upon optic nerve regeneration in zebrafish, we examined the spatiotemporal distribution of phosphorylated ribosomal protein S6 (pS6), a downstream effector of mTOR, on retinal cross sections at various times after optic nerve injury (Figure 1). In naïve, uninjured zebrafish retinae, pS6 immunostaining was detected only in a few cells of the INL (Figure 1A). In comparison, S6 phosphorylation was quickly, although transiently induced in acetylated tubulin-positive RGCs upon optic nerve crush (Figures 1A,B,D). At 2 days post injury (dpi), $78.1 \pm 3.8 \%$ RGCs were strongly positive for pS6, whereas hardly any pS6 signal was detected in RGCs at time points beyond 4 dpi (Figure 1D). Interestingly, injury-induced mTOR activation was also observed in cholinergic amacrines identified by co-staining with antibodies against cholinergic acetyl transferase (CHAT) in the INL (Figures 1A,C,D). However, pS6 immunoreactivity in amacrines was only significantly increased at 4-16 dpi. Thus, optic nerve injury induced mTOR activation in RGCs and amacrine cells with different time courses.

\section{mTOR Activity is Induced in Cultured RGCs}

To test whether mTOR is also activated in axotomized RGCs in vitro, we co-stained dissociated retinal cultures from naïve zebrafish with pS6 and acetylated tubulin antibodies at various time points. At $2 \mathrm{~h}$ in culture, already $36.7 \pm$ 2.5\% RGCs were pS6-positive (Figure 2A). This proportion rose to $50.8 \pm 1.9 \%$ by $6 \mathrm{~h}$ and stayed at this level for 2 days before significantly declining at 4 days in culture (25.7 $\pm 2.4 \%)$. Therefore, mTOR activity was similarly induced in axotomized RGCs in culture and in vivo. The faster onset and lower overall penetrance ( $\sim 50$ vs. $\sim 80 \%$; compare Figures 1D, 2A) is likely attributable to the cell culture conditions.

To assess whether mTOR activity could be modulated by cytokines similar to mammalian RGCs (Park et al., 2008; Leibinger et al., 2012), some retinal cultures were concurrently treated with CNTF. CNTF has recently been shown to promote neurite growth of zebrafish RGCs (Elsaeidi et al., 2014; Diekmann et al., 2015) and indeed significantly increased the percentage of pS6-positive RGCs at 2 and $6 \mathrm{~h}$ compared to controls $(52.6 \pm 2 \%$ and $62.8 \pm 2.1 \%$, respectively) (Figures $2 \mathrm{~A}, \mathbf{B}$ ). However, no CNTF-induced increase of mTOR activity was observed at 1, 2 and 4 days in culture (Figure 2A). Interestingly,
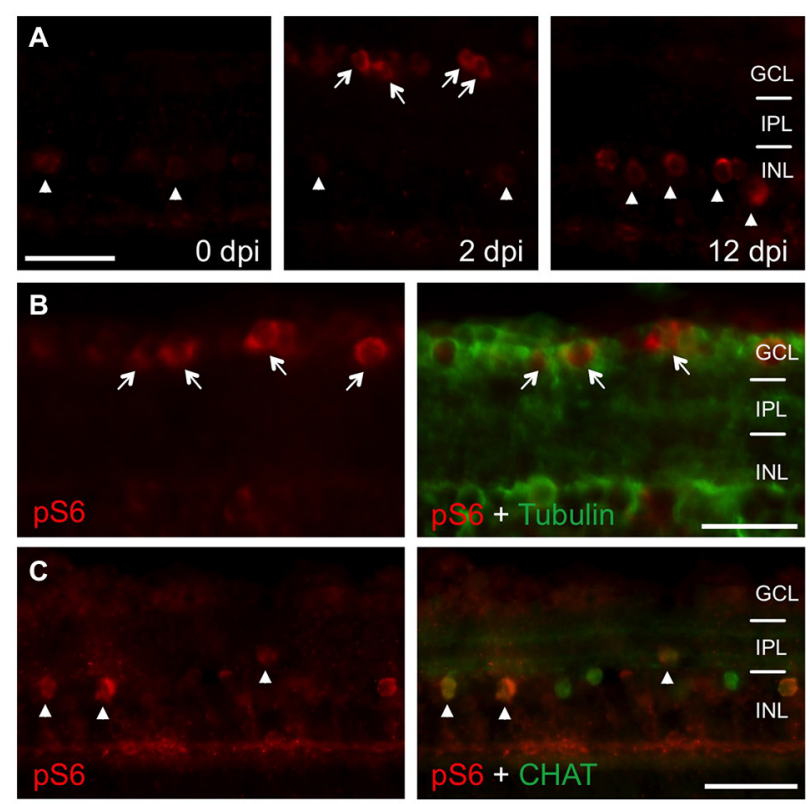

D 90

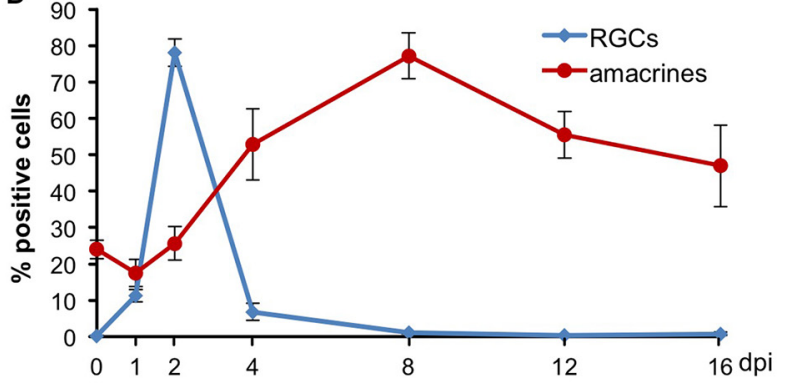

FIGURE 1 | Retinal mTOR activation upon optic nerve injury. (A) Immunohistochemistry of phosphorylated S6 (pS6) on retinal cross sections at 0,2 and 12 days post injury (dpi) indicates induction of mTOR activity in the ganglion cell (GCL, arrows) and inner nuclear (INL, arrowheads) layers after optic nerve axotomy. (B) Co-immunostaining of a retinal cross section (2 dpi) with pS6 (red) and acetylated tubulin (green) antibodies identifies pS6-positive cells as retinal ganglion cells (RGCs; arrows). (C) Co-immunostaining of a retinal cross section ( $0 \mathrm{dpi}$ ) with pS6 (red) and choline acetyl transferase (CHAT, green) antibodies identifies cholinergic amacrine cells (arrowheads). (D) Time course of pS6-positive RGCs (blue) and amacrines (red) after optic nerve crush. Data represent means \pm SEM of two independent experiments. $\mathrm{GCL}=$ ganglion cell layer; $\mathrm{IPL}=$ inner plexiform layer; INL = inner nuclear layer. Scale bar $=25 \mu \mathrm{m}$.

addition of CNTF to retinal cultures at 1 day post dissociation could similarly not promote increased pS6 levels (data not shown), indicating insensitivity to $\mathrm{CNTF}$ at later incubation times.

We also validated that mTOR activation was indeed the underlying cause of S6 phosphorylation in zebrafish RGCs. Application of the potent mTOR inhibitor rapamycin abolished S6 phosphorylation in RGCs, independent of CNTF treatment (Figures 2B,C). Conversely, the PTEN inhibitor bpV(phen), which promotes PI3K and subsequently mTOR activity (Posner et al., 1994; Schmid et al., 2004), significantly increased the percentage of pS6-positive RGCs either alone or in combination with CNTF compared to untreated controls 

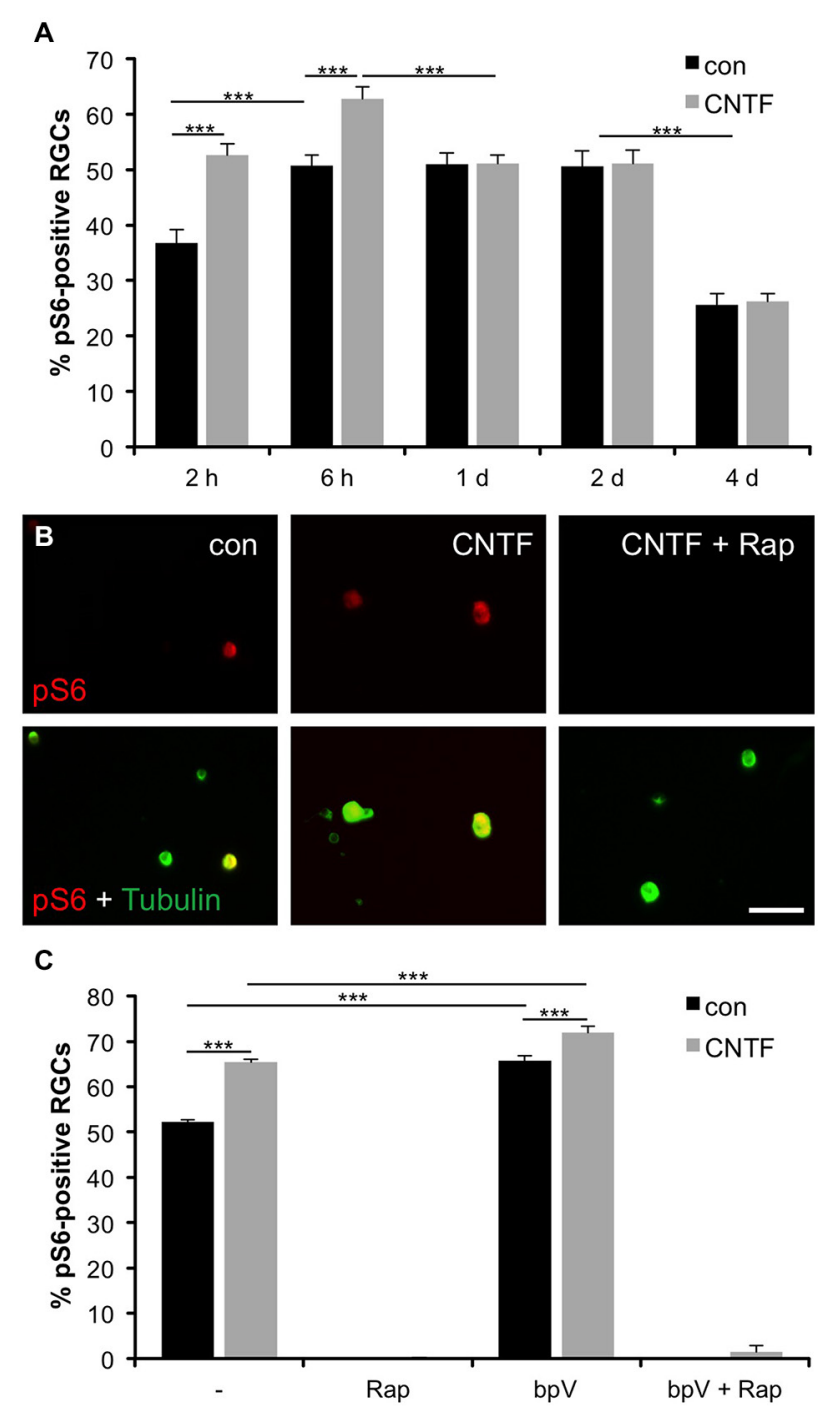

FIGURE 2 | Induction of mTOR activity in cultured RGCs.

(A) Quantification of the percentage of retinal ganglion cells (RGCs) positive for phosphorylated S6 (pS6) at 2 and 6 hours (h) as well as 1, 2 and 4 days (d) in culture with or without ciliary neurotrophic factor (CNTF; $1 \mathrm{ng} / \mathrm{ml}$ ) treatment. Data represent means \pm SEM of three independent experiments, each with three repeats. Treatment effects: $p<0.001$. (B) Representative pictures of cultured RGCs stained with pS6 (red) and acetylated tubulin (green) antibodies at $6 \mathrm{~h}$ in culture. Cells were either treated with vehicle (con), CNTF $(1 \mathrm{ng} / \mathrm{ml})$ or CNTF + rapamycin (Rap; $10 \mathrm{nM})$. Scale bar $=50 \mu \mathrm{m}$. (C) Percentage of pS6-positive RGCs at $6 \mathrm{~h}$ in culture after treatment with either vehicle (-), rapamycin (Rap; $10 \mathrm{nM}$ ), PTEN inhibitor bisperoxovanadium (bpV(phen); $10 \mathrm{nM}$ ), or bpV(phen) + Rap. Data represent means \pm SEM of at least two independent experiments, each with three repeats. Treatment effects: $p<0.001$.

$(65.7 \pm 1.1 \%(\mathrm{bpV})$ and $71.9 \pm 1.4 \%(\mathrm{bpV}+\mathrm{CNTF})$ vs. $52.2 \pm 0.4 \%$ (control); Figure 2C). Similar to CNTF treatment, $\mathrm{bpV}$ (phen) was unable to increase mTOR activity upon application at 1 day in culture (data not shown). Concurrent $\mathrm{bpV}$ (phen) + rapamycin treatment again annulled S6 phosphorylation (Figure 2C), indicating that bpV(phen) acts on S6 through increasing mTOR activity. Overall, the extent of
S6 phosphorylation correlates with mTOR activity in zebrafish RGCs.

\section{Induction of Neurite Growth Correlates with mTOR Activity}

In an effort to evaluate whether mTOR activity is required for axonal growth, we next measured neurites of isolated RGCs grown in the presence of rapamycin (mTOR inhibition) or $\mathrm{bpV}$ (phen) (mTOR activation). Interestingly, rapamycin concentrations abolishing S6 phosphorylation (Figures 2B,C) did not affect neurite growth per se [72.4 $\pm 1.9 \mu \mathrm{m}$ (Rap) vs. $68.0 \pm 0.8 \mu \mathrm{m}$ (untreated control)]. However, treatment with either CNTF or bpV(phen), which increased mTOR activity to comparable extents, significantly increased neurite growth of dissociated RGCs $(82.0 \pm 1.3 \mu \mathrm{m}$ and $85.6 \pm 2.2 \mu \mathrm{m}$, respectively; Figures 3A,B). Moreover, combination of CNTF and $\mathrm{bpV}$ (phen), which increased mTOR activity the most (Figure 2C), also showed longer outgrowth than each treatment alone $(97.6 \pm 3.8 \mu \mathrm{m})$. These growth-promoting effects of CNTF and $\mathrm{bpV}$ (phen) were abrogated in the presence of rapamycin $[69.4 \pm 1.4 \mu \mathrm{m}(\mathrm{CNTF}+\mathrm{Rap}), 71.1 \pm 0.4 \mu \mathrm{m}$ (bpV + Rap) and $70.5 \pm 1.3 \mu \mathrm{m}(\mathrm{CNTF}+\mathrm{bpV}+\mathrm{Rap})]$, indicating a correlation between increased mTOR activity and PI3K/AKT-stimulated neurite growth in culture (Figure 3B). Thus, mTOR activity is seemingly not required for basal axon growth, but enhanced
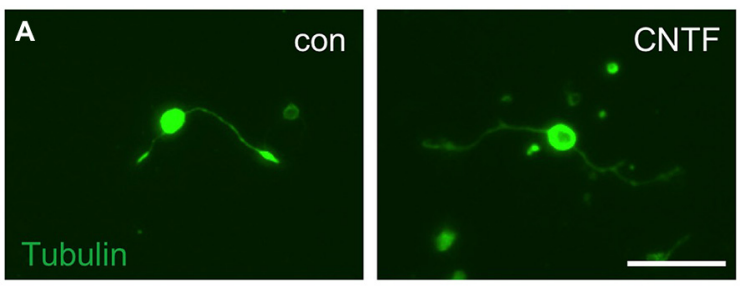

B

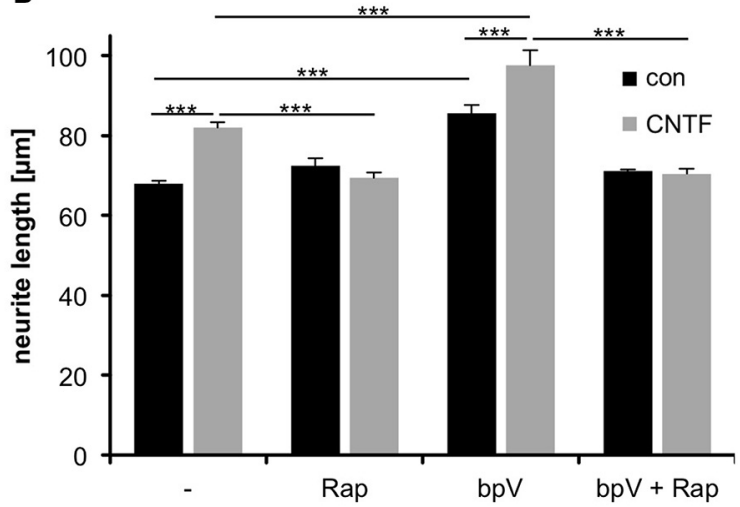

FIGURE 3 | Correlation of mTOR activity with CNTF- and PTEN inhibitor-induced neurite growth of cultured RGCs. (A) Representative pictures of cultured retinal ganglion cells (RGCs) stained with acetylated tubulin antibody at 4 days in culture and exposure to vehicle (con) and ciliary neurotrophic factor (CNTF, $1 \mathrm{ng} / \mathrm{ml})$, respectively. Scale bar $=50 \mu \mathrm{m}$.

(B) Quantification of average RGC neurite length at 4 days in culture after treatment with either vehicle control (-), rapamycin (Rap; $10 \mathrm{nM}$ ), PTEN inhibitor bpV(phen) (10 nM), or bpV(phen) + Rap. Data represent means \pm SEM of at least two independent experiments, each with three repeats. Treatment effects: $p<0.001$. 
levels are sufficient to promote further growth of RGC neurites in culture.

\section{Inhibition of mTOR Activity Compromises Axon Regeneration and Functional Recovery}

In an attempt to investigate the relevance of mTOR activity for axonal regeneration, we first explored pharmacological approaches for efficient mTOR inhibition in vivo. In our hands, neither intravitreal nor intraperitoneal injections of rapamycin yielded consistent, longer-term down-regulation of retinal S6 phosphorylation (data not shown). Therefore, zebrafish were placed directly into either DMSO- or rapamycin-containing water immediately after optic nerve crush, as similar treatment has previously been shown to impede mTOR induction upon fin amputation (Hirose et al., 2014). Using this approach, mTOR activation was efficiently inhibited as verified by abolished pS6 levels on retinal cross-sections (Figure 4). Whereas S6 phosphorylation was strongly induced in RGCs of control retinae at $2 \mathrm{dpi}$ (Figure 4A) and in cholinergic amacrines at $6 \mathrm{dpi}$ (Figure 4C), no pS6 staining was detected in rapamycin-treated zebrafish at these time points (Figures 4B,D). Therefore, mTOR activity also underlies increased S6 phosphorylation upon optic nerve injury in vivo and can be prevented longer-term by supplementing the fish water with rapamycin.

To investigate whether inhibition of mTOR activity would impact axon regeneration upon optic nerve injury, we took advantage of our recently established protocol that enables the accurate analysis of regenerating axons in wholemount optic nerve preparations using GAP43:GFP transgenic zebrafish (Diekmann et al., 2015). As previously described, quite a few severed axons had already grown past the lesion site back into the injured nerve at $2.5 \mathrm{dpi}$ in control animals (Figures $\mathbf{5} \mathbf{A}, \mathbf{A}^{\prime}$ ). In comparison, rapamycin treatment reduced the number and/or length of regenerating axons as quantified at two different sites posterior to the lesion (Figures $\mathbf{5 B}, \mathbf{B}^{\prime}, \mathbf{C}$ ). Similarly, pixel intensities were reduced in optic nerves of rapamycin-treated zebrafish at 5 dpi (data not shown). Systemic rapamycin treatment has previously been described to reduce GAP43 expression in peripheral, but not optic nerves in mice (Abe et al., 2010; Leibinger et al., 2012). Therefore, we next addressed the possibility that the observed reduction of regenerating axons was rather based on impaired GFP expression. To this end, we compared transgenic gfp and endogenous gap43 mRNA expression levels in vehicle- and rapamycin-treated retinae by quantitative RT-PCR (Figure 5D). In comparison to control animals, neither gfp nor gap43 expression was significantly changed at 2 dpi in rapamycin-treated retinae, thereby excluding this possibility.

Finally, we investigated whether rapamycin-induced impairment of axonal regeneration would have functional consequences. As reported previously, zebrafish swim slightly oblique towards the side of the intact eye upon unilateral optic nerve crush (Figure 5E). This tilt lessens progressively with ongoing axonal regeneration (Lindsey and Powers, 2007; Mensinger and Powers, 2007). Thus, the swimming position can be used as an indicator for functional recovery of vision. In the vehicle-treated control group, significant improvements were initially detected at $11 \mathrm{dpi}\left(7.2^{\circ}\right.$ vs. $10.9^{\circ}$ at $\left.1 \mathrm{dpi}\right)$ and the swimming angle lessened to $4.2^{\circ}$ at $18 \mathrm{dpi}$. In comparison, rapamycin-treated zebrafish swam in a significantly more tilted position from $11 \mathrm{dpi}\left(10.1^{\circ}\right)$ up to $18 \mathrm{dpi}\left(6.5^{\circ}\right.$; Figure $\left.5 \mathrm{~F}\right)$. Thus, impairment of mTOR activity upon optic nerve injury
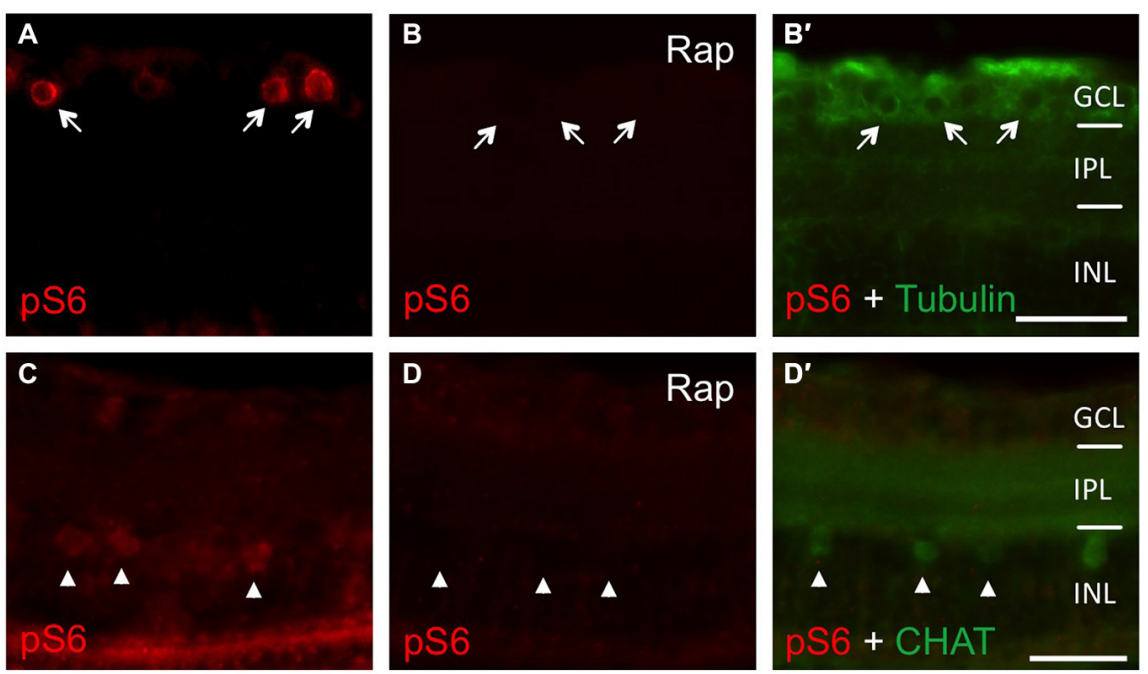

FIGURE 4 | Efficient inhibition of mTOR activity in zebrafish in vivo. (A, B, B') Immunostaining of retinal cross sections with phosphorylated S6 (pS6, red) and acetylated tubulin (green) antibodies at 2 days post injury. Fish were either treated with DMSO (A) or $0.2 \mu \mathrm{M}$ rapamycin (Rap, B) prior to tissue preparation. Rapamycin treatment abrogated pS6 staining in retinal ganglion cells (RGCs; arrows). (C,D,D') Immunostaining of retinal cross sections with pS6 (red) and choline acetyl transferase (CHAT; green) antibodies at 6 days post injury. Fish were either treated with DMSO (C) or $0.2 \mu \mathrm{M}$ rapamycin (Rap, D) prior to tissue preparation. Rapamycin treatment abolished pS6 staining in amacrines (arrowheads). $\mathrm{GCL}=$ ganglion cell layer; $\mathrm{IPL}=$ inner plexiform layer; $\mathrm{INL}=$ inner nuclear layer; Scale bar $=25 \mu \mathrm{m}$. 

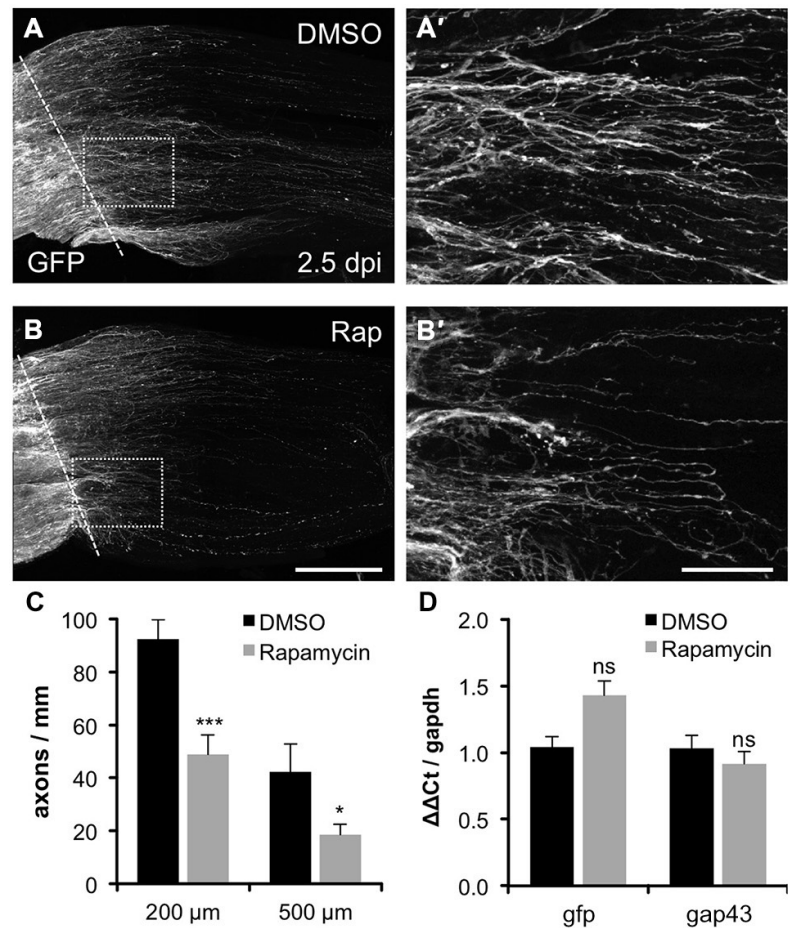

D
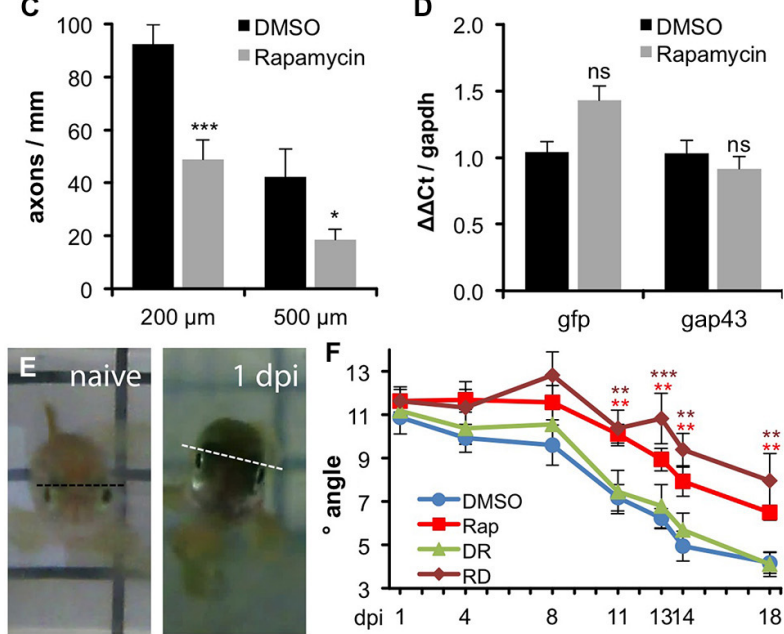

FIGURE 5 | Compromised axon regeneration and functional recovery upon mTOR inhibition. (A,B) Maximum intensity projections $(85 \times 0.9 \mu \mathrm{m}$ confocal z-sections) of wholemount optic nerves from DMSO- (A) and $0.2 \mu \mathrm{M}$ rapamycin (Rap, B) -treated GAP43::GFP zebrafish at 2.5 days post injury (dpi). The lesion site is indicated with a dashed line, proximal is to the left. Scale bar $=200 \mu \mathrm{m}$. ( $\left.\mathbf{A}^{\prime}, \mathbf{B}^{\prime}\right)$ Higher magnifications of the boxed areas in (A) and (B), respectively, using maximum intensity projections of $10 \times 0.9 \mu \mathrm{m}$ confocal stacks. Scale bar $=50 \mu \mathrm{m}$. (C) Quantification of axon profiles per $\mathrm{mm}$ optic nerve diameter on single z-sections at 200 and $500 \mu \mathrm{m}$ posterior to the lesion site of DMSO- and rapamycin-treated zebrafish, respectively (for details see "Materials and Methods" Section). Data represent means \pm SEM of 8 optic nerves from two independent experiments. Treatment effects compared to DMSO control: ${ }^{\star \star \star} p<0.001 ;{ }^{*} p<0.05$ (D) Quantitative real-time PCR for green fluorescent protein (gfp) and growth associated protein 43 (gap43) in relation to glyceraldehyde-3-phosphate dehydrogenase (gapdh) in retinae isolated from zebrafish 2 days post injury that were treated either with vehicle (DMSO) or $0.2 \mu \mathrm{M}$ rapamycin (Rap), respectively. Data represent mean $\Delta \Delta \mathrm{Ct} \pm \mathrm{SEM}$ of at least three different fish per experimental group. ns = non-significant (E) Representative pictures of the swimming position of a naïve zebrafish and a fish 1 day post unilateral right optic nerve crush (1 dpi), respectively. (F) Quantification of the oblique swimming position of DMSO (blue)- and rapamycin (Rap; red)-treated zebrafish at 1, 4, 11, 13, 14 and 18 days post injury (dpi). In addition, some zebrafish received rapamycin-treatment only during the first 3 days of the experiment $(R D$; brown). Another group was initially held in DMSO (0-3 dpi) and then transferred to rapamycin for the remainder of the experiment (DR; green). Data represent means \pm SEM of at least five zebrafish per group. Treatment effects compared to DMSO control: ${ }^{\star \star \star} p<0.001 ;{ }^{\star \star} p<0.01$. significantly compromised axonal regeneration and functional recovery. Interestingly, reduced regeneration was also observed upon temporary rapamycin treatment early after optic nerve crush (0-3 dpi), correlating with increased mTOR activity in RGCs (Figure 5F). On the other hand, late rapamycin application (4-18 dpi) had no effect on visual recovery, indicating that the delay of functional recovery was caused by abolished mTOR activation in RGCs within the first days after injury and that mTOR induction in cholinergic amacrines at later time points is negligible for optic nerve regeneration. Overall, mTOR signaling seems not absolutely essential for axonal regeneration, as rapamycin could only delay, rather than completely block axon growth and functional recovery. Nevertheless, the initial burst of mTOR activity upon optic nerve injury, which is fundamentally different to its regulation in mammals, supports regenerative processes in regeneration-competent zebrafish.

\section{Discussion}

The current study characterizes the relevance of active mTOR for axonal regrowth and functional recovery after optic nerve injury in a regeneration-competent CNS model. Indeed, mTOR activity was markedly, although temporarily induced in zebrafish RGCs upon optic nerve injury in vivo as well as in dissociated neuronal cell cultures. However, mTOR activity was not essential for axonal growth per se, but correlated with cytokine- and PTEN inhibitor-induced neurite extension in vitro. Moreover, inhibition of mTOR using rapamycin significantly reduced axonal regeneration in vivo and compromised functional recovery after optic nerve injury. Thus, regulation of mTOR activity after axonal injury in regeneration-competent zebrafish seems fundamentally different from the non-regenerating mammalian CNS. Nevertheless, active mTOR contributes to axon regeneration in both systems.

Induction of mTOR in zebrafish retina upon optic nerve injury was monitored using the phosphorylation levels of ribosomal protein S6 as described previously (Park et al., 2008; Leibinger et al., 2012). S6 is phosphorylated by S6K1, which itself is a target of mTOR. Accordingly, rapamycin as an established mTOR inhibitor diminished pS6 levels in retinal cell cultures as well as in vivo. In addition, stimulation of mTOR through the PI3K/AKT pathway with either the cytokine CNTF or the PTEN inhibitor bpV(phen) consistently elevated pS6 levels in dissociated RGCs. Therefore, the phosphorylation status of S6 can be used as a faithful readout of mTOR activity in zebrafish retinae.

The progression of mTOR activity after optic nerve injury in zebrafish differed significantly from the one in rodents. In retinae from naïve, adult mice, only $10-15 \%$ of RGCs contain high levels of phosphorylated S6 and reportedly have the highest probability to regenerate (Duan et al., 2015). This proportion is even further reduced upon optic nerve injury (Park et al., 2008; Leibinger et al., 2012). In contrast, no pS6 staining was observed in RGCs of naïve zebrafish retinae. However, S6 phosphorylation in RGCs was markedly induced in vivo shortly after optic nerve injury as well as in dissociated cell 
cultures. In addition, prolonged mTOR induction was observed in cholinergic amacrines after an initial lag phase. The activity of mTOR is reportedly controlled by the PI3K/AKT, MAPK/ERK, CDK42/phopholipase D and wnt pathways (Fang et al., 2001, 2003; Ma et al., 2005; Huang and Manning, 2009; Hirose et al., 2014). Interestingly, leukemia inhibitory factor (LIF), a IL6like cytokine, which reportedly activates the PI3K/AKT/mTOR pathway in rodents ( $\mathrm{Li}$ et al., 2014), is strongly upregulated in zebrafish RGCs early after axotomy (Ogai et al., 2014) and could potentially contribute to the transient mTOR activation (Figure 6). However, the elucidation of the detailed stimuli involved in activating mTOR after optic nerve injury in zebrafish awaits further investigations.

The burst of mTOR activity in RGCs shortly after optic nerve injury was quickly attenuated, as hardly any pS6-positive RGCs were detected at $4 \mathrm{dpi}$. This again approximately coincides with the attenuation of LIF expression in zebrafish (Ogai et al., 2014). In addition, activated mTOR itself has been shown to strongly repress the PI3K/AKT pathway upstream of PI3K, thereby forming a negative feedback loop (Harrington et al., 2005; Manning and Cantley, 2007). Furthermore, S6K1mediated phosphorylation of Rictor inhibits mTORC2 and AKT signaling (Julien and Roux, 2010). Both of these auto-regulatory mechanisms could be involved in the observed mTOR downregulation in zebrafish RGCs (Figure 6A). Interestingly, mTOR activity could not be induced by CNTF or PTEN inhibitor application at later time points in culture, indicating potential deactivation of this signaling pathway during later regenerative processes. For this reason, augmentation of in vivo mTOR activity with the PTEN inhibitor in an attempt to experimentally increase axon regeneration is envisaged impractical.

Overall, the observed time course of S6 phosphorylation matches the regulation of mTOR activity after injury in another regeneration competent model, the mammalian sciatic nerve. Two-fold increased pS6 levels were detected in dorsal root ganglion (DRG) neurons at $1 \mathrm{dpi}$ that returned to basal levels

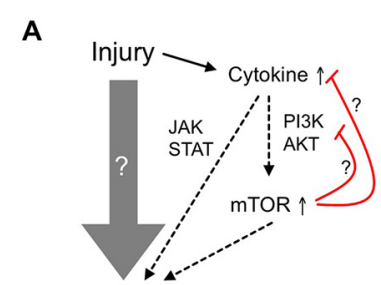

Axon growth

Regeneration
B

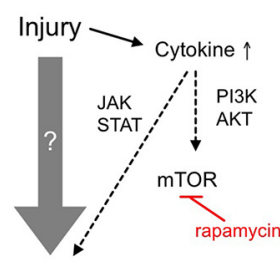

reduced axon growth delayed regeneration
FIGURE 6 | Schematic showing the ancillary contribution of mTOR activity to axon regeneration in adult zebrafish. (A) Upon optic nerve injury, diverse and prevalently unknown molecular mechanisms (gray arrow) are activated in adult zebrafish RGCs to enable axon growth and functional regeneration. Among others, cytokines such as LIF are released and activate the JAK/STAT and PI3K/AKT/mTOR pathways that contribute to axon regeneration. The initial burst of mTOR activity could then be quickly attenuated by negative feedback loops (red lines). (B) Inhibition of mTOR using rapamycin only partially reduces axon regrowth and compromises functional recovery, suggesting an ancillary rather than essential role of mTOR activity in zebrafish optic nerve regeneration. by 4 dpi (Abe et al., 2010). Comparably, S6K phosphorylation was also transiently induced in regenerating blastemal cells $6 \mathrm{~h}$ to 3 days after zebrafish fin amputation (Hirose et al., 2014), consistently indicating short bursts of mTOR activity in regenerating tissues. In contrast, impaired regeneration in the mammalian CNS is associated with reduced mTOR activity in mature neurons, which is further decreased upon axonal injury (Park et al., 2008; Leibinger et al., 2012). Therefore, mTOR regulation in regeneration-competent (mammalian PNS, zebrafish) and -incompetent (mammalian CNS) systems seems fundamentally different and it would be interesting to analyze the effect of a comparable course of mTOR activity (short burst of strong mTOR activity in all RGCs followed by quick down-regulation) on the regenerative ability of mammalian CNS neurons.

Similar to mouse RGCs, basal neurite growth in cell culture was not affected by rapamycin treatment in zebrafish, as pS6negative RGCs grew neurites equally well (Leibinger et al., 2012 and this study). On the other hand, mTOR activity closely correlated with PI3K/AKT-induced axonal growth of zebrafish RGCs. Consistent with previous reports (Elsaeidi et al., 2014; Diekmann et al., 2015), application of the cytokine CNTF or the PTEN-inhibitor pbV(phen) increased pS6 levels as well as neurite length in culture and this growth-promotion was abrogated in the presence of rapamycin. In mice RGCs, on the contrary, rapamycin could only compromise the neurite growth promoting effect of CNTF on myelin substrate, but not on laminin (Leibinger et al., 2012). The underlying cause for these disparate observations might be founded on the non-inhibitory nature of the fish optic nerve (Wanner et al., 1995). Similar to its effect in culture, rapamycin reduced, but not completely blocked axonal regrowth in vivo (Figure 6B). Remarkably, LIF induction after axotomy follows a similar time course as mTOR activation and functional recovery after optic nerve injury is similarly delayed upon LIF knockdown (Ogai et al., 2014). Therefore, it is quite conceivable that the partial inhibition of axonal regeneration upon rapamycin treatment reflects impaired PI3K/AKT/mTOR signaling, which, consistent with our in vitro results, would depend on LIF or other cytokine release shortly after axotomy (Figure 6B). Further experiments need to address this possibility in the future. As mTOR activity is quickly down-regulated after a short burst, it would be interesting to analyze whether prolonged activation of this pathway would lead to enhanced axon regeneration or whether the observed deactivation is essentially required for later regenerative processes. Due to the likely presence of intrinsic negative feedback loops within this pathway (Figure 6), these experiments might require inducible, RGC-specific expression of constitutively active mTOR mutants. Unfortunately, this approach is currently rather impractical in adult zebrafish.

Maintenance of pre-injury mTOR activity levels in injured mammalian RGCs, for instance by RGC-specific genetic deletion of PTEN, markedly promotes in vivo axon regeneration (Park et al., 2008; Smith et al., 2009). Likewise, constitutive activation of mTOR upon genetic deletion of TSC1 potently enhances axon regeneration of RGCs and DRG neurons 
(Park et al., 2008; Abe et al., 2010). On the other hand, the number of regenerating mouse RGC axons upon inflammatory stimulation was reduced by rapamycin treatment only at long distances, whereas the extent of short axons remained unaffected (Leibinger et al., 2012). These findings were interpreted in a way that mTOR is not essentially required for the initial switch of neurons into an active regenerative state. However, increased mTOR activity is seemingly important to maintain the regenerative state, potentially due to enhanced protein and lipid biosynthesis. In zebrafish, axon regeneration after optic nerve crush was significantly compromised, but not completely inhibited by rapamycin, suggesting a comparable ancillary role of $\mathrm{mTOR}$ in this regeneration-competent species.

Obviously, systemic rapamycin treatment could inhibit mTOR activity in cell types other than RGCs. For example, mTOR signaling has been shown to regulate oligodendrocyte differentiation and CNS myelination (Narayanan et al., 2009; Tyler et al., 2009) and the positive effects of rapamycin treatment after contusive spinal cord injury have been partly attributed to reduced secondary neural tissue damage due to suppression of macrophage/microglia activation and abrogation of reactive astrogliosis (Kanno et al., 2012). In addition, it is now known that chronic rapamycin treatment could inhibit mTORC2 just as the classic target mTORC1, thereby potentially affecting cytoskeleton dynamics (Sarbassov et al., 2006). However, it is currently unknown whether these processes/cell types might be involved in optic nerve regeneration in zebrafish. Nevertheless, the conclusion of a rather RGC-specific effect of our results is supported by the time course of mTOR activation and the differential functional recovery upon timed rapamycin treatment. Axon regeneration was only compromised in zebrafish receiving rapamycin at early time points after optic nerve crush (0-3 dpi), when mTOR was markedly

\section{References}

Abe, N., Borson, S. H., Gambello, M. J., Wang, F., and Cavalli, V. (2010). Mammalian target of rapamycin (mTOR) activation increases axonal growth capacity of injured peripheral nerves. J. Biol. Chem. 285, 28034-28043. doi: 10. 1074/jbc.M110.125336

Becker, C. G., and Becker, T. (2007). Growth and pathfinding of regenerating axons in the optic projection of adult fish. J. Neurosci. Res. 85, 2793-2799. doi: $10.1002 /$ jnr.21121

Bernhardt, R. R., Tongiorgi, E., Anzini, P., and Schachner, M. (1996). Increased expression of specific recognition molecules by retinal ganglion cells and by optic pathway glia accompanies the successful regeneration of retinal axons in adult zebrafish. J. Comp. Neurol. 376, 253-264. doi: 10.1002/(sici)1096-9861(19961209)376:2<253::aid-cne7>3. $0 . \operatorname{co} ; 2-2$

Bernhardt, R. R. (1999). Cellular and molecular bases of axonal regeneration in the fish central nervous system. Exp. Neurol. 157, 223-240. doi: 10.1006/exnr.1999. 7059

Bormann, P., Roth, L. W., Andel, D., Ackermann, M., and Reinhard, E. (1999). zfNLRR, a novel leucine-rich repeat protein is preferentially expressed during regeneration in zebrafish. Mol. Cell. Neurosci. 13, 167-179. doi: 10.1006/mcne. 1999.0742

Cai, D., Qiu, J., Cao, Z., McAtee, M., Bregman, B. S., and Filbin, M. T. (2001). Neuronal cyclic AMP controls the developmental loss in ability of axons to regenerate. J. Neurosci. 21, 4731-4739. induced in RGCs, whereas delayed rapamycin treatment (4-18 dpi) had no effect. Therefore, the induction of mTOR in cholinergic amacrines does not seem to contribute to optic nerve regeneration. Similarly, the observed reduction of regenerating axons upon rapamycin treatment could not be ascribed to impaired GFP marker expression. In contrast to mTORdependent induction of GAP43 in injured rodent sciatic nerves (Abe et al., 2010), no reduction of GAP43 promoterdriven gfp transcription was detected in zebrafish retinae upon rapamycin treatment. This result is in accordance with data showing no influence of rapamycin on the expression of GAP43 and other regeneration-associated genes in rodent retinae after inflammatory stimulation (Leibinger et al., 2012) and again indicates that inhibition of mTOR activity does not generally impair the transformation of RGCs into a regenerative state.

In conclusion, the current study demonstrates the differential regulation of mTOR activity in CNS regeneration-competent vs. -incompetent models. Although not required for basal axon growth, our data suggest that the early burst of mTOR activity still contributes to the positive regenerative outcome in zebrafish. Therefore, further mechanistic insights into functional axon regeneration are provided, highlighting that, at least in fish, other signaling pathways than mTOR are more crucially involved in CNS axon regeneration.

\section{Acknowledgments}

We thank Anastasia Andreadaki and Marcel Kohlhaas, University of Düsseldorf for technical support. We are very grateful to Dr. Udvadia, University of Wisconsin, for providing $\operatorname{Tg}$ (fgap43:GFP) zebrafish. This work was funded by a research grant of the medical faculty of the Heinrich Heine University.

Christie, K. J., Webber, C. A., Martinez, J. A., Singh, B., and Zochodne, D. W. (2010). PTEN inhibition to facilitate intrinsic regenerative outgrowth of adult peripheral axons. J. Neurosci. 30, 9306-9315. doi: 10.1523/JNEUROSCI.627109.2010

Diekmann, H., and Fischer, D. (2013). Glaucoma and optic nerve repair. Cell Tissue Res. 353, 327-337. doi: 10.1007/s00441-013-1596-8

Diekmann, H., Kalbhen, P., and Fischer, D. (2015). Characterization of optic nerve regeneration using transgenic zebrafish. Front. Cell. Neurosci. 9:118. doi: 10. 3389/fncel.2015.00118

Duan, X., Qiao, M., Bei, F., Kim, I. J., He, Z., and Sanes, J. R. (2015). Subtypespecific regeneration of retinal ganglion cells following axotomy: effects of osteopontin and mTOR signaling. Neuron 85, 1244-1256. doi: 10.1016/j. neuron.2015.02.017

Elsaeidi, F., Bemben, M. A., Zhao, X. F., and Goldman, D. (2014). Jak/Stat signaling stimulates zebrafish optic nerve regeneration and overcomes the inhibitory actions of Socs3 and Sfpq. J. Neurosci. 34, 2632-2644. doi: 10. 1523/JNEUROSCI.3898-13.2014

Fang, Y., Park, I. H., Wu, A. L., Du, G., Huang, P., Frohman, M. A., et al. (2003). PLD1 regulates mTOR signaling and mediates Cdc42 activation of S6K1. Curr. Biol. 13, 2037-2044. doi: 10.1016/j.cub.2003.11.021

Fang, Y., Vilella-Bach, M., Bachmann, R., Flanigan, A., and Chen, J. (2001). Phosphatidic acid-mediated mitogenic activation of mTOR signaling. Science 294, 1942-1945. doi: 10.1126/science. 1066015 
Fischer, D., and Leibinger, M. (2012). Promoting optic nerve regeneration. Prog. Retin. Eye Res. 31, 688-701. doi: 10.1016/j.preteyeres.2012. 06.005

Fischer, D., Pavlidis, M., and Thanos, S. (2000). Cataractogenic lens injury prevents traumatic ganglion cell death and promotes axonal regeneration both in vivo and in culture. Invest. Ophthalmol. Vis. Sci. 41, 3943-3954.

Gao, Y., Deng, K., Hou, J., Bryson, J. B., Barco, A., Nikulina, E., et al. (2004). Activated CREB is sufficient to overcome inhibitors in myelin and promote spinal axon regeneration in vivo. Neuron 44, 609-621. doi: 10.1016/j.neuron. 2004.10.030

Goldberg, J. L., Klassen, M. P., Hua, Y., and Barres, B. A. (2002). Amacrinesignaled loss of intrinsic axon growth ability by retinal ganglion cells. Science 296, 1860-1864. doi: 10.1126/science.1068428

Harrington, L. S., Findlay, G. M., and Lamb, R. F. (2005). Restraining PI3K: mTOR signalling goes back to the membrane. Trends Biochem. Sci. 30, 35-42. doi: 10. 1016/j.tibs.2004.11.003

Hauk, T. G., Leibinger, M., Müller, A., Andreadaki, A., Knippschild, U., and Fischer, D. (2010). Stimulation of axon regeneration in the mature optic nerve by intravitreal application of the toll-like receptor 2 agonist Pam3Cys. Invest. Ophthalmol. Vis. Sci. 51, 459-464. doi: 10.1167/iovs.09-4203

Hirose, K., Shiomi, T., Hozumi, S., and Kikuchi, Y. (2014). Mechanistic target of rapamycin complex 1 signaling regulates cell proliferation, cell survival and differentiation in regenerating zebrafish fins. BMC Dev. Biol. 14:42. doi: 10 . 1186/s12861-014-0042-9

Huang, J., and Manning, B. D. (2009). A complex interplay between Akt, TSC2 and the two mTOR complexes. Biochem. Soc. Trans 37, 217-222. doi: 10. 1042/bst0370217

Julien, L. A., and Roux, P. P. (2010). mTOR, the mammalian target of rapamycin. Med. Sci. (Paris) 26, 1056-1060. doi: 10.1051/medsci/201026121056

Kanno, H., Ozawa, H., Sekiguchi, A., Yamaya, S., Tateda, S., Yahata, K., et al. (2012). The role of mTOR signaling pathway in spinal cord injury. Cell Cycle 11, 3175-3179. doi: 10.4161/cc.21262

Laplante, M., and Sabatini, D. M. (2009). mTOR signaling at a glance. J. Cell Sci. 122, 3589-3594. doi: 10.1242/jcs.051011

Leaver, S. G., Cui, Q., Bernard, O., and Harvey, A. R. (2006). Cooperative effects of bcl-2 and AAV-mediated expression of CNTF on retinal ganglion cell survival and axonal regeneration in adult transgenic mice. Eur. J. Neurosci. 24, 3323-3332. doi: 10.1111/j.1460-9568.2006. 05230.x

Leibinger, M., Andreadaki, A., and Fischer, D. (2012). Role of mTOR in neuroprotection and axon regeneration after inflammatory stimulation. Neurobiol. Dis. 46, 314-324. doi: 10.1016/j.nbd.2012.01.004

Leibinger, M., Müller, A., Andreadaki, A., Hauk, T. G., Kirsch, M., and Fischer, D. (2009). Neuroprotective and axon growth-promoting effects following inflammatory stimulation on mature retinal ganglion cells in mice depend on ciliary neurotrophic factor and leukemia inhibitory factor. J. Neurosci. 29, 14334-14341. doi: 10.1523/JNEUROSCI. 2770-09.2009

Leibinger, M., Müller, A., Gobrecht, P., Diekmann, H., Andreadaki, A., and Fischer, D. (2013). Interleukin-6 contributes to CNS axon regeneration upon inflammatory stimulation. Cell Death Dis. 4:e609. doi: 10.1038/cddis.2013.126

Li, X., Yang, Q., Yu, H., Wu, L., Zhao, Y., Zhang, C., et al. (2014). LIF promotes tumorigenesis and metastasis of breast cancer through the AKTmTOR pathway. Oncotarget 5, 788-801.

Lindsey, A. E., and Powers, M. K. (2007). Visual behavior of adult goldfish with regenerating retina. Vis. Neurosci. 24, 247-255. doi: 10. 1017/s0952523806230207

Lingor, P., Tönges, L., Pieper, N., Bermel, C., Barski, E., Planchamp, V., et al. (2008). ROCK inhibition and CNTF interact on intrinsic signalling pathways and differentially regulate survival and regeneration in retinal ganglion cells. Brain 131, 250-263. doi: 10.1093/brain/awm284

Liu, Q., and Londraville, R. L. (2003). Using the adult zebrafish visual system to study cadherin-2 expression during central nervous system regeneration. Methods Cell Sci. 25, 71-78. doi: 10.1023/b:mics.0000006854. 18378.fC

Liu, K., Lu, Y., Lee, J. K., Samara, R., Willenberg, R., Sears-Kraxberger, I., et al. (2010). PTEN deletion enhances the regenerative ability of adult corticospinal neurons. Nat. Neurosci. 13, 1075-1081. doi: 10.1038/nn.2603
Livak, K. J., and Schmittgen, T. D. (2001). Analysis of relative gene expression data using real-time quantitative PCR and the 2(-Delta Delta C(T)) Method. Methods 25, 402-408. doi: 10.1006/meth.2001. 1262

Ma, L., Chen, Z., Erdjument-Bromage, H., Tempst, P., and Pandolfi, P. P. (2005). Phosphorylation and functional inactivation of TSC2 by Erk implications for tuberous sclerosis and cancer pathogenesis. Cell 121, 179-193. doi: 10.1016/j. cell.2005.02.031

Maiese, K., Chong, Z. Z., Shang, Y. C., and Wang, S. (2013). mTOR: on target for novel therapeutic strategies in the nervous system. Trends Mol. Med. 19, 51-60. doi: 10.1016/j.molmed.2012.11.001

Manning, B. D., and Cantley, L. C. (2007). AKT/PKB signaling: navigating downstream. Cell 129, 1261-1274. doi: 10.1016/j.cell.2007.06.009

McDowell, A. L., Dixon, L. J., Houchins, J. D., and Bilotta, J. (2004). Visual processing of the zebrafish optic tectum before and after optic nerve damage. Vis. Neurosci. 21, 97-106. doi: 10.1017/s0952523804 043019

Mensinger, A. F., and Powers, M. K. (2007). Visual function in regenerating teleost retina following surgical lesioning. Vis. Neurosci. 24, 299-307. doi: 10. $1017 /$ s0952523807070265

Müller, A., Hauk, T. G., Leibinger, M., Marienfeld, R., and Fischer, D. (2009). Exogenous CNTF stimulates axon regeneration of retinal ganglion cells partially via endogenous CNTF. Mol. Cell. Neurosci. 41, 233-246. doi: 10.1016/j. mcn.2009.03.002

Narayanan, S. P., Flores, A. I., Wang, F., and Macklin, W. B. (2009). Akt signals through the mammalian target of rapamycin pathway to regulate CNS myelination. J. Neurosci. 29, 6860-6870. doi: 10.1523/JNEUROSCI.0232-09. 2009

Ning, K., Drepper, C., Valori, C. F., Ahsan, M., Wyles, M., Higginbottom, A., et al. (2010). PTEN depletion rescues axonal growth defect and improves survival in SMN-deficient motor neurons. Hum. Mol. Genet. 19, 3159-3168. doi: 10. 1093/hmg/ddq226

Ogai, K., Kuwana, A., Hisano, S., Nagashima, M., Koriyama, Y., Sugitani, K., et al. (2014). Upregulation of leukemia inhibitory factor (LIF) during the early stage of optic nerve regeneration in zebrafish. PLoS One 9:e106010. doi: 10. 1371/journal.pone.0106010

Park, K. K., Liu, K., Hu, Y., Smith, P. D., Wang, C., Cai, B., et al. (2008). Promoting axon regeneration in the adult CNS by modulation of the PTEN/mTOR pathway. Science 322, 963-966. doi: 10.1126/science. 1161566

Pernet, V., Joly, S., Dalkara, D., Jordi, N., Schwarz, O., Christ, F., et al. (2013). Long-distance axonal regeneration induced by CNTF gene transfer is impaired by axonal misguidance in the injured adult optic nerve. Neurobiol. Dis. 51, 202-213. doi: 10.1016/j.nbd.2012.11.011

Posner, B. I., Faure, R., Burgess, J. W., Bevan, A. P., Lachance, D., ZhangSun, G., et al. (1994). Peroxovanadium compounds. A new class of potent phosphotyrosine phosphatase inhibitors which are insulin mimetics. J. Biol. Chem. 269, 4596-4604.

Sarbassov, D. D., Ali, S. M., Sengupta, S., Sheen, J. H., Hsu, P. P., Bagley, A. F., et al. (2006). Prolonged rapamycin treatment inhibits mTORC2 assembly and Akt/PKB. Mol. Cell 22, 159-168. doi: 10.1016/j.molcel.2006. 03.029

Schmid, A. C., Byrne, R. D., Vilar, R., and Woscholski, R. (2004). Bisperoxovanadium compounds are potent PTEN inhibitors. FEBS Lett. 566, 35-38. doi: 10.1016/s0014-5793(04)00435-1

Schwab, M. E., and Bartholdi, D. (1996). Degeneration and regeneration of axons in the lesioned spinal cord. Physiol. Rev. 76, 319-370.

Smith, P. D., Sun, F., Park, K. K., Cai, B., Wang, C., Kuwako, K., et al. (2009). SOCS3 deletion promotes optic nerve regeneration in vivo. Neuron 64, 617-623. doi: 10.1016/j.neuron.2009.11.021

Stuermer, C. A., Bastmeyer, M., Bähr, M., Strobel, G., and Paschke, K. (1992). Trying to understand axonal regeneration in the CNS of fish. J. Neurobiol. 23, 537-550. doi: 10.1002/neu.480230508

Tyler, W. A., Gangoli, N., Gokina, P., Kim, H. A., Covey, M., Levison, S. W., et al. (2009). Activation of the mammalian target of rapamycin (mTOR) is essential for oligodendrocyte differentiation. J. Neurosci. 29, 6367-6378. doi: 10.1523/JNEUROSCI.0234-09. 2009 
Udvadia, A. J. (2008). $3.6 \mathrm{~kb}$ genomic sequence from Takifugu capable of promoting axon growth-associated gene expression in developing and regenerating zebrafish neurons. Gene Expr. Patterns 8, 382-388. doi: 10.1016/j. gep.2008.05.002

Wanner, M., Lang, D. M., Bandtlow, C. E., Schwab, M. E., Bastmeyer, M., and Stuermer, C. A. (1995). Reevaluation of the growth-permissive substrate properties of goldfish optic nerve myelin and myelin proteins. J. Neurosci. 15, 7500-7508.

Westerfield, M. (1989). The zebrafish book: a guide for the laboratory use of zebrafish (Brachydanio rerio). Eugene: University of Oregon.
Conflict of Interest Statement: The authors declare that the research was conducted in the absence of any commercial or financial relationships that could be construed as a potential conflict of interest.

Copyright (c) 2015 Diekmann, Kalbhen and Fischer. This is an open-access article distributed under the terms of the Creative Commons Attribution License (CC BY). The use, distribution and reproduction in other forums is permitted, provided the original author(s) or licensor are credited and that the original publication in this journal is cited, in accordance with accepted academic practice. No use, distribution or reproduction is permitted which does not comply with these terms. 